$54^{\text {ème }}$ Congrès de la SFMBCB, 03009 (2011)

DOI: $10.1051 / \mathrm{sfmbcb} / 20115403009$

(C) Owned by the authors, published by EDP Sciences, 2011

\title{
A propos d'un cas de pemphigoïde cicatricielle des muqueuses
}

\author{
Martinez J, Cousty S, L'Homme A, Courtois B, Duran D \\ ${ }^{1}$ UFR d'Odontologie, Université Paul Sabatier, Toulouse III, France \\ ju.bwi@hotmail.fr
}

La pemphigoide cicatricielle des muqueuses constitue un groupe de maladies bulleuses sousépithéliales, chroniques, auto-immunes, inflammatoires, affectant de façon prédominante les muqueuses. Elle est caractérisée par un dépôt linéaire d'IgG, d'IgA et de C3, le long de la membrane basale (Chan 2002) et atteint surtout les femmes dans leur cinquième ou sixième décennie (Scully 2007). Bénigne dans la majorité des cas, elle peut parfois être mortelle lors d'une atteinte cutanéo-muqueuse étendue (Scully 2005, Chan 2002).

Le cas rapporté est celui d'une patiente de 93 ans qui consulte pour une lésion palatine gênant le port de sa prothèse complète supérieure, évoluant depuis plus de 2 ans. La patiente a déjà consulté plusieurs praticiens qui ont prescrit des traitements inadaptés (application topique d'acide trichloracétique, aciclovir...).

L'examen clinique montre des ulcérations palatines sur une muqueuse érythémateuse, très douloureuse et saignant volontiers au contact. Le «signe de la pince » est positif en périphérie des lésions. Il existe également une desquamation gingivale importante. Il n'y a aucune atteinte cutanée associée, ni ophtalmique ou gynécologique.

L'examen histologique montre un décollement sous-épithélial ; l'immunofluorescence directe un dépôt linéaire de complexes immuns le long de la jonction dermo-épidermique, caractérisé par la présence d'IgG, d'IgA et de facteurs du complément. En immunofluorescence indirecte, aucun anticorps circulant n'est retrouvé.

Le diagnostic de pemphigoïde cicatricielle des muqueuses est retenu. Une corticothérapie topique à base de propionate de clobetasol à $0,05 \%$ est initiée. La patiente est revue régulièrement pour suivre l'évolution des lésions. Après six mois, les lésions ont régressé de façon significative et le port de la prothèse est possible sans douleur.

Le diagnostic de pemphigoïde cicatricielle des muqueuses est basé sur l'observation minutieuse des lésions et de leur localisation (uniquement muqueuse ou cutanéo-muqueuse). L'examen histologique et en immunofluorescence directe sont nécessaires pour confirmer le diagnostic clinique. L'immunofluorescence indirecte n'est positive que dans 20\% des cas (Casiglia 2001). Au travers du cas clinique présenté, la chronologie de la démarche diagnostique est détaillée. C'est cette dernière qui, pondérée et réfléchie, peut éviter au patient une errance diagnostique ainsi que des traitements inappropriés pendant plusieurs années.

This is an Open Access article distributed under the terms of the Creative Commons Attribution-Noncommercial License 3.0, which permits unrestricted use, distribution, and reproduction in any noncommercial medium, provided the original work is properly cited. 